Received: 2016.12 .03

Accepted: 2017.01 .24

Published: 2017.10 .20

Authors' Contribution:
A Study Design
B Data Collection
C Statistical Analysis
D Data Interpretation
E Manuscript Preparation
F Literature Search
G Funds Collection

\title{
Poland Syndrome with Ipsilateral Hypoplasia of Gluteal Muscles and Contralateral Hidradenitis Suppurativa
}

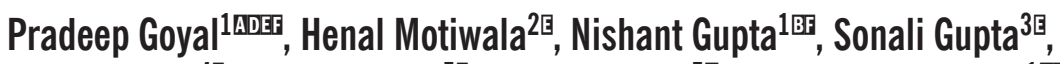 \\ Itisha Bansa ${ }^{4 \rrbracket}$, Kusum Hooda ${ }^{5 匹}$, Yogesh Kumar ${ }^{50}$, Thomas D. Olsavsky ${ }^{1 \mathrm{GGEB}}$ \\ ${ }^{1}$ Department of Radiology, Saint Vincent's Medical Center, Bridgeport, CT, U.S.A. \\ ${ }^{2}$ Frank H. Netter M.D. School of Medicine at Quinnipiac University, North Haven, CT, U.S.A. \\ ${ }^{3}$ Department of Medicine, Saint Vincent's Medical Center, Bridgeport, CT, U.S.A. \\ ${ }^{4}$ Department of Anesthesiology, New York Methodist Hospital, Brooklyn, NY, U.S.A. \\ ${ }^{5}$ Department of Radiology, Yale New Haven Health at Bridgeport Hospital, Bridgeport, CT, U.S.A.
}

Author's address: Pradeep Goyal, Department of Radiology and Imaging, Saint Vincent's Medical Center, 2800 Main Street, Bridgeport, CT 06606, U.SA., e-mail: pradeepgoyal78@gmail.com

\section{Summary}

Background: $\quad$ Poland syndrome (PS) is a rare congenital anomaly associated with absent or hypoplastic pectoralis major muscle and a wide spectrum of ipsilateral thoracic and upper extremity deformities. Hidradenitis suppurativa (HS) is a recurrent inflammatory follicular disease that commonly affects the apocrine-bearing skin and involves follicular occlusion and hyperkeratosis.

Case Report: $\quad$ We report a case of a 46-year-old male with a history of chronic recurrent hidradenitis suppurativa who was incidentally found to have a simple type of Poland syndrome with incidental hypoplasia of ipsilateral gluteal muscles.

Conclusions: Not only can PS present with HS, which we describe for the first time, but it is also associated with a wide variability of symptoms such as previously unknown co-existence of gluteal muscles hypoplasia.

MeSH Keywords: $\quad$ Congenital Abnormalities • Hidradenitis Suppurativa • Poland Syndrome

PDF file: $\quad$ http://www.polradiol.com/abstract/index/idArt/902677

\section{Background}

Poland syndrome (PS) is a rare congenital anomaly that was first reported in 1841 by Alfred Poland who described a case of a 19-year-old medical student from London [1]. Absence or hypotrophy of the pectoralis major muscle is always present in PS, either as an isolated finding or in combination with other symptoms, such as defects of the thoracic wall and adjacent limbs. There are two forms of PS - a complex form and a simple form. The complex form presents early and is associated with multiple chest wall and limb anomalies. The simple form is usually diagnosed later in life and presents with chest wall asymmetry without associated limb anomalies.

The incidence of PS is one in 30,000-32,000 live births, with a male-to- female ratio of 2-3: 1 [2].
There are few hypotheses for the etiopathogenesis of PS. Vascular etiology, such as "subclavian artery supply disruption sequence", was proposed as a possible link between a number of birth defects including Poland syndrome, Klippel-Feil syndrome, Möbius syndrome, and Sprengel's deformity [3]. Another hypothesis puts forward a disruption of the embryonic lateral plate/para-axial mesoderm between the $16^{\text {th }}$ and $28^{\text {th }}$ day after fertilization [4]. However, both of the above-mentioned theories cannot explain the wide clinical variability, familial occurrence, or presence of bilateral symptoms. Para-dominant inheritance of somatic mutation appears to be the only possible mechanism that can explain such features $[5,6]$. Somatic mosaicism is associated with a specific class of non-inherited genetic diseases; somatic mosaicism is characterized by the fact that mutations are found only in a proportion of cells, and such mutations could be lethal, if they were present in all embryonic cells. In this way, mosaicism would 


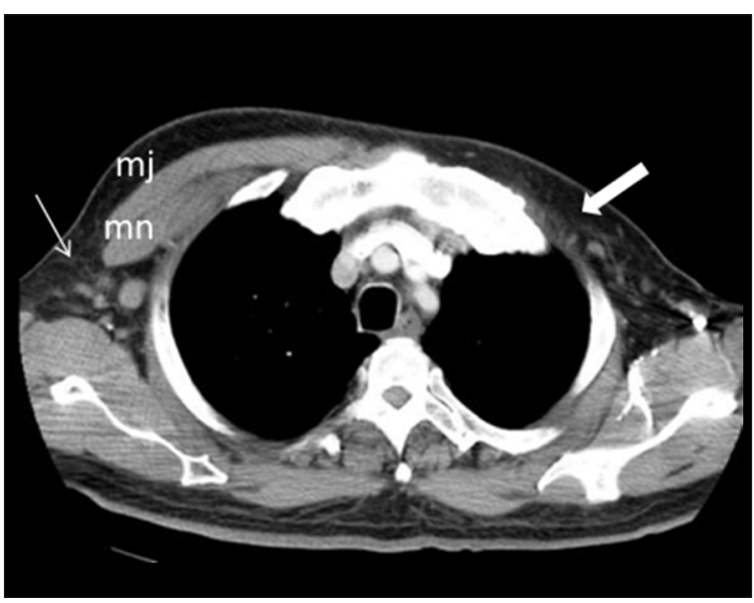

Figure 1. Contrast-enhanced CT of the chest demonstrates features of Poland syndrome: asymmetrically reduced subcutaneous fat layer, absence of the pectoralis major (mj) and minor (mn) muscles in the left chest wall, (thick arrow) and minimal fat stranding (thin arrow) in the right axilla and right lateral chest wall.

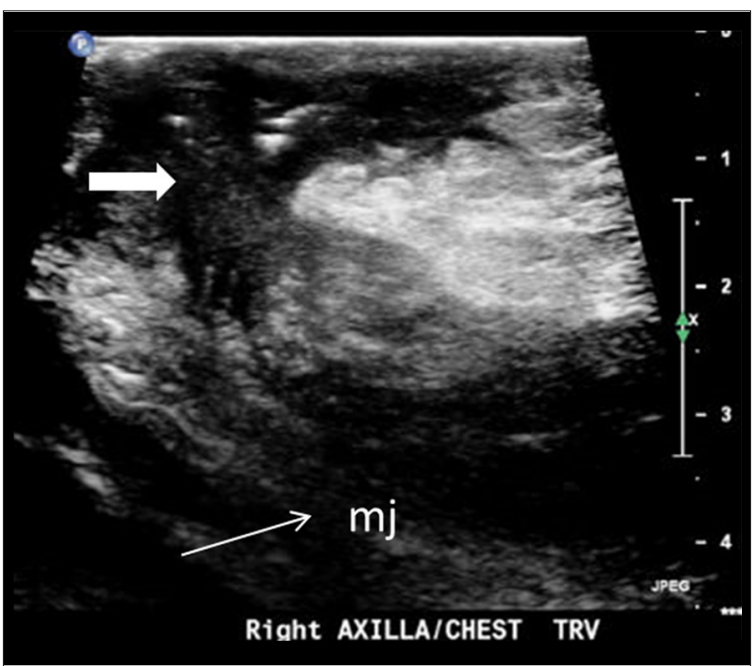

Figure 2. US of the chest demonstrates a complex collection in (thick arrow) the subcutaneous tissue of the right axilla/chest wall extending to pectoralis muscles (mj) (arrow).

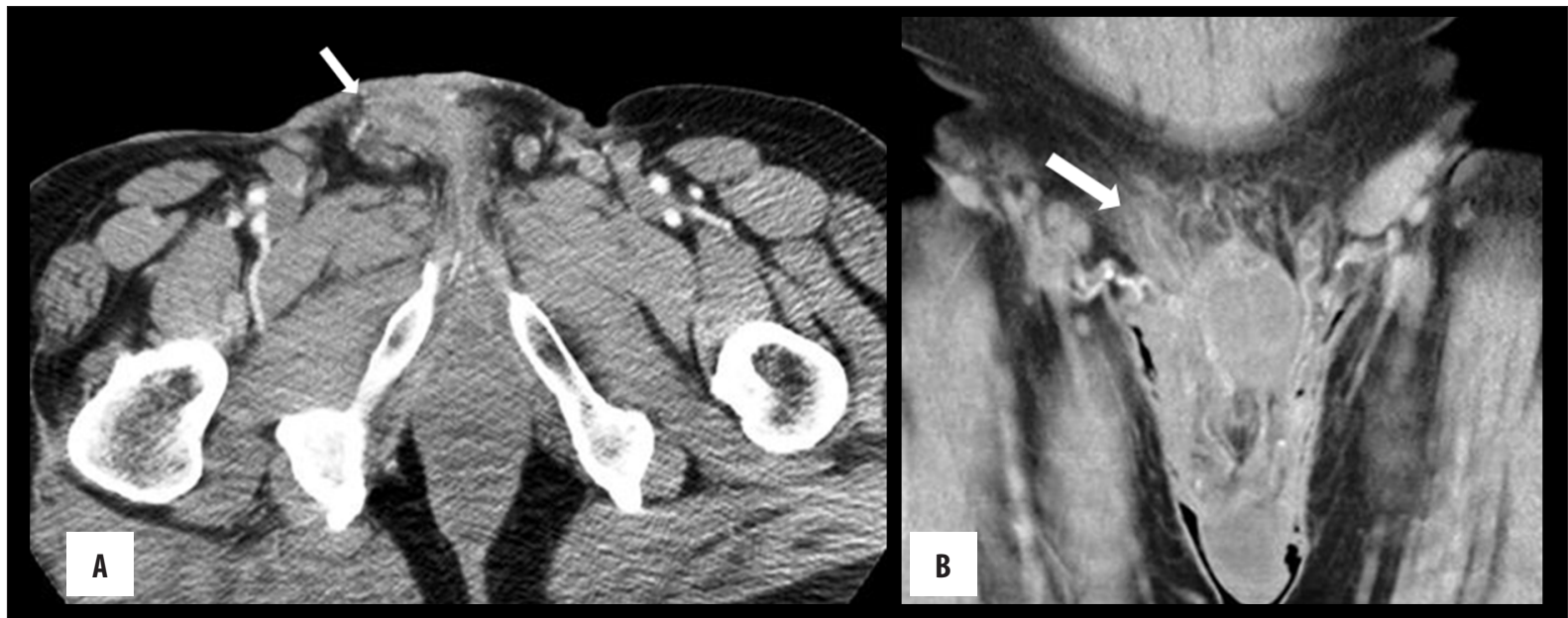

Figure 3. CT of the abdomen and pelvis demonstrates features of hidradenitis suppurativa: (A) axial image demonstrates a thick-walled, lowattenuation collection in the subcutaneous tissues of the right penile base, with thickening of the overlying skin; (B) coronal image demonstrates abnormally enhancing soft tissue infiltration in the subcutaneous fat of the right penile base (arrow), reactive hyperemia on the left penile base, and bilateral inguinal lymph nodes.

reduce tissue hypoplasia or aplasia resulting from the presence of a lethal mutation in a limb bud cell line, as the cells non-affected by the mutation would continue to develop normally. The extent of deformity in mosaicism depends on the timing of mutations. Late mutations produce only localized skin and soft tissue anomalies, whereas early mutations may result in severe chest wall and limb defects. Genetic counseling is not standardized for PS, as it is difficult to assess recurrence risk due to sporadic and familial occurrence.

HS is a chronic, inflammatory, recurrent, debilitating follicular disease of the skin that usually presents after puberty with painful, deep seated, inflamed lesions in the apocrine gland-bearing areas of the body, most commonly in the axillary, inguinal, and anogenital regions [7]. HS is one element of the 'follicular occlusion tetrad', along with acne conglobata, pilonidal cysts, and dissecting cellulitis, all of which share a similar pathogenesis [7]. However, the exact etiology of HS remains unknown. Smoking, overweight/obesity, and metabolic syndrome (DM type 2, hypertension, and hyperlipidemia) are strongly associated with HS and may exacerbate it [8]. Complications associated with HS include but are not limited to anal, urethral, and rectal strictures or fistulas, lymphedema, increased incidence of cancers, and arthritis [8,9].

\section{Case Report}

A 46-year-old man presented with complaints of heaviness in the right axillary/chest wall region and drainage and pain in the right groin. The medical history was remarkable for chronic recurrent right groin HS, uncontrolled type-2 diabetes mellitus (DM), hypertension, hyperlipidemia, alcohol abuse, and smoking. CT of the chest demonstrated asymmetric reduction of the subcutaneous fat 


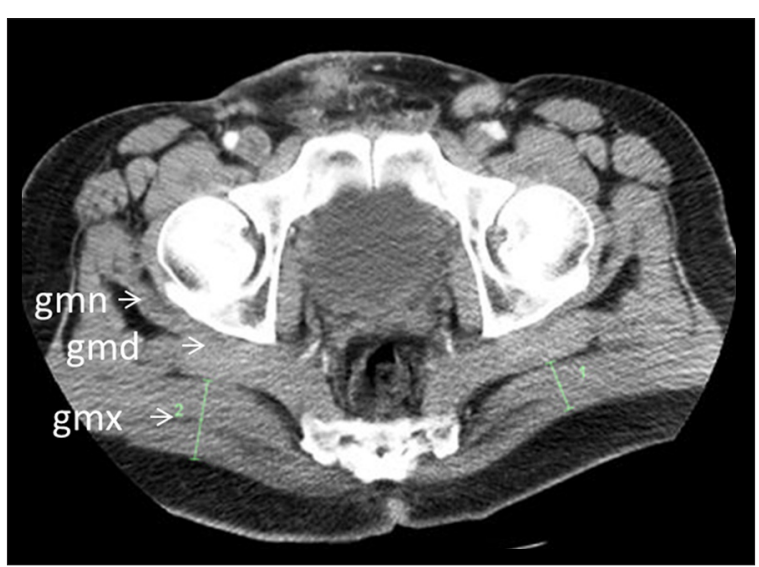

Figure 4. Contrast-enhanced CT of the pelvis demonstrates hypoplastic left gluteal muscles (compared to the right side). Note that the difference between the left (1) and right (2) gluteus maximus (gmx) muscles is obvious, whereas the differences between gluteus medius (gmd) and gluteus minimus (gmn) muscles are subtle.

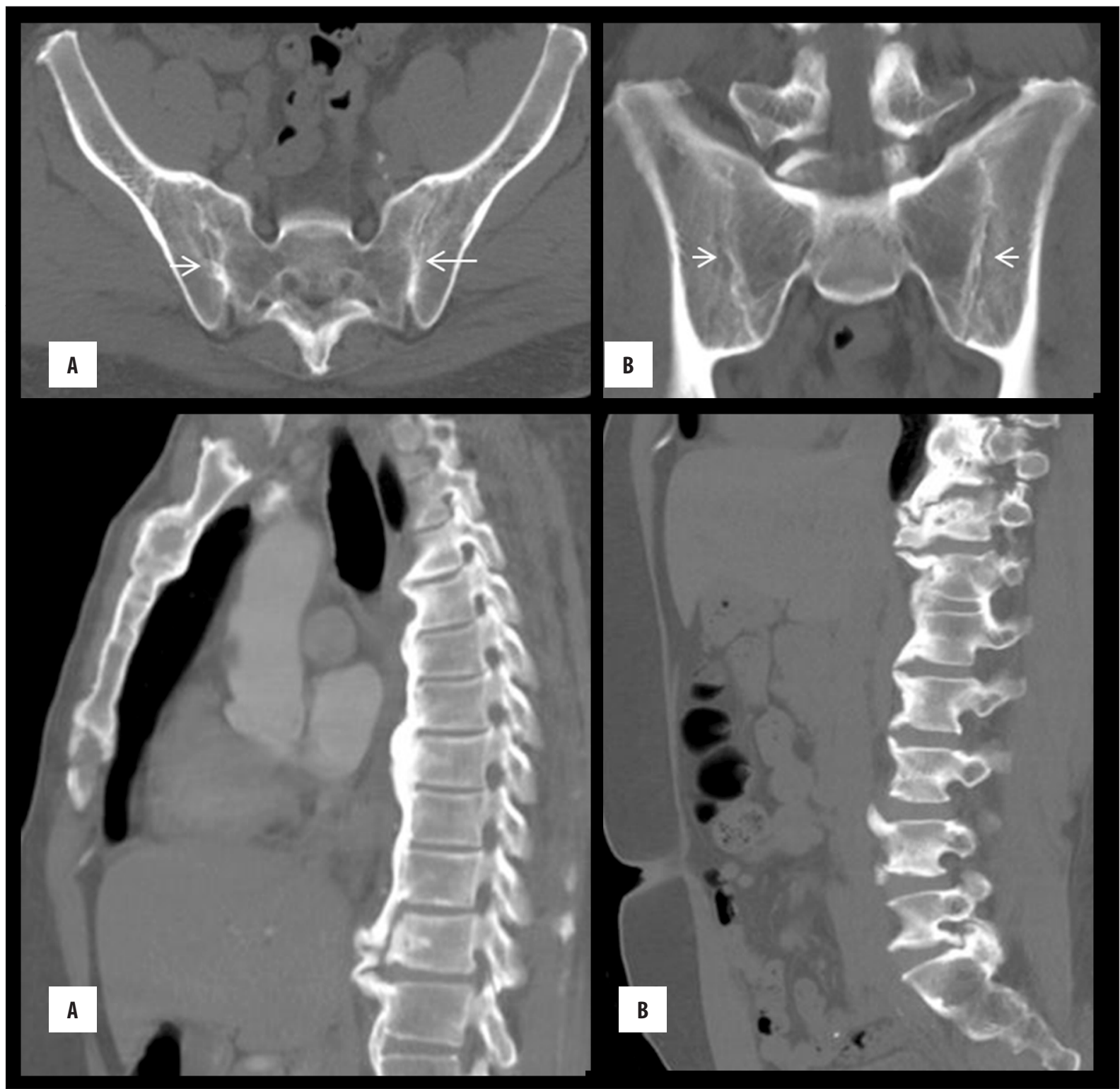

Figure 5. CT of the pelvis and spine demonstrates features of seronegative spondyloarthropathy in a patient with hidradenitis suppurativa. (T of the pelvis - axial (A) and coronal (B) images demonstrate asymmetric (left>right) ankyloses in the sacroiliac joints. CT of the thoracic spine (C) and lumbar spine (D) demonstrates marginal syndesmophytes. 
incisions and drainage of the recurrent right groin HS. Approximately 1 year after the diagnosis of PS, the patient developed right axillary/chest abscess. US of the chest demonstrated a complex collection in the subcutaneous tissue of the right axilla/chest wall that was extending to the pectoralis muscles (Figure 2). The patient underwent incision and drainage, with debridement of the deep abscess of the axilla and was started on Adalimumab. Prior abdominal and pelvic CT of the patient demonstrated persistent, thickwalled, low-attenuation collection in the subcutaneous tissues of the right penile base, with abnormally enhancing soft tissue infiltration in the adjacent fat and overlying skin thickening (Figure 3). Hypoplasia of the left gluteal maximus muscle was found incidentally (Figure 4). Also, there were asymmetric ankyloses in the sacroiliac joints (Figure 5A, 5B) and multilevel non-marginal syndesmophytes (Figure 5C, 5D), findings that are associated with seronegative spondyloarthropathy.

\section{Discussion}

Poland syndrome (PS) was first described in 1841 by an English surgeon, Alfred Poland, in a 19-year-old medical student from London [1]. PS is a spectrum of rare congenital malformations that present with at least absence or hypoplasia of the pectoralis major muscle. Other manifestations include thoracic wall and adjacent limb defects. Two forms of PS have been described in the literature [1], a simple form and a complex form. The more common simple form is characterized by the absence of pectoralis major muscle, a small and displaced nipple, and small bones of the upper extremity [1]. The less common complex form is characterized by absence of the pectoralis major muscle and other musculoskeletal malformations of the thorax, such as absent or hypoplastic serratus anterior muscle, shortened clavicle, rib deformities, frequent severe shortening of the upper extremity, and webbing of the fingers [1]. Our patient had the simple form of PS, with absent pectoralis major and minor muscles without associated limb anomalies; however, we incidentally found hypoplasia of the gluteal muscles.

Hidradenitis suppurativa (HS) is a recurrent painful inflammatory disease manifested by subcutaneous abscesses, sinus tracts, and scarring that predominantly arise in the apocrine gland-bearing skin [6]. The differential diagnosis for HS is extensive (Table 1), but HS can easily be differentiated from other diseases by the appearance of the lesions, post-pubertal age of onset, characteristic locations, resistance to antibiotics, recovery of multiple species (rather than a single species) of bacteria on culture, absence of fever, and the lack of significant laboratory findings. Crohn's disease is both a differential diagnosis and an associated disease. Cutaneous Crohn's disease must be differentiated from HS in its perianal location by necessary workups. Diagnosis of HS in our case was initially made because of the presence of recurrent abscesses, sinus tract, and scars in the groin. Our patient had all the major risk factors of HS, such as smoking, obesity, and metabolic syndrome. Moreover, the patient developed seronegative spondyloarthropathy, one of the complications of long-standing HS.
Table 1. Differential diagnosis of hidradenitis suppurativa.

\begin{tabular}{ll}
\hline \multicolumn{1}{c}{ Early lesions } & \multicolumn{1}{c}{ Late lesions } \\
\hline Common abscess & Actinomycosis \\
\hline Carbuncles & Anal fistula \\
\hline Cutaneous blastomycosis & Crohn's disease \\
\hline Furunculosis & Developmental fistulae \\
\hline Infected Bartholin's gland & Granuloma inguinale \\
\hline $\begin{array}{l}\text { Infected or inflamed epidermal } \\
\text { cysts }\end{array}$ & Ischiorectal abscess \\
\hline Nodular Acne & Lymphogranuloma venereum \\
\hline Pilonidal cyst & Scrofuloderma \\
\hline
\end{tabular}

Poland syndrome has been associated with an increased risk of several cancers, including but not limited to leukemia, non-Hodgkin lymphoma, and breast cancers [10]. Also, squamous cell carcinoma can develop in long-standing HS [8]. Therefore, we counseled our patient for long-term surveillance due to an increased overall cancer risk.

Poland syndrome has also been associated with several dermatologic conditions, but its association with HS has not been described earlier. Hyperhidrosis is one of the triggers for HS flares. PS is commonly associated with anhidrosis due to the absence of sweat glands in the axillary and/or mammary areas [4].The presence of HS in the right axilla in our patient may suggest compensatory hyperhidrosis in response to anhidrosis in the left axilla. Our patient also had hypoplasia of ipsilateral (left) gluteal muscles, which has never been described in the literature. Perhaps, it remains unnoticed due to the absence of any obvious deformity, or it is just coincidental. However, the presence of recurrent HS on the contralateral (right) groin suggests compensatory hyperhidrosis in the right groin. However, the observed degree of hypoplasia is less apparent in the gluteal muscles. This may be in part related to disuse atrophy of the right gluteal muscles secondary to chronic relapsing $\mathrm{HS}$ on the right side. This observation suggests that ipsilateral hypoplasia of gluteal muscles is not just coincidental and needs further assessment in the already published cases of Poland's syndrome.

A wide variability of symptoms, such as gluteal muscles involvement in our patient, familial occurrence, and presence of bilateral symptoms in PS [2] can only be explained by the theory of somatic mosaicism $[5,6]$. Somatic mosaicism is characterized by the fact that mutations are found only in a proportion of cells, and they could be lethal, if they were present in all embryonic cells. So far, molecular evidence for this hypothesis is lacking, despite recent advances in the detection of somatic mosaicism. For instance, many diseases of overgrowth and vascular malformations, such as Proteus syndrome, CLOVES syndrome, Klippel-Trenaunay syndrome, Sturge-Weber syndrome, Maffucci syndrome, and Ollier disease are caused by somatic mosaicism, and the particular somatic mutations have been described. A limited number of molecular 
pathways seem to be involved in these disorders, some of which are also implicated in cancer [11].

\section{Conclusions}

Not only can PS present with HS, which we describe for the first time, but it is also associated with a wide variability of symptoms such as previously unknown co-existence of gluteal muscles hypoplasia. Considering the proposed mechanism of somatic mosaicism, the case presented in this article can provoke discussions on the etiopathogenesis of Poland syndrome.

\section{Conflict of interest}

The authors declare that they have no conflict of interest.

\section{References:}

1. Seyfer AE, Fox JP, Hamilton CG: Poland syndrome: Evaluation and treatment of the chest wall in 63 patients. Plast Reconstr Surg, 2010; 126(3): 902-11

2. Baban A, Torre M, Costanzo S et al: Familial Poland anomaly revisited. Am J Med Genet Part A, 2012; 158(1), 140-49

3. Bavinck JNB, Weaver DD: Subclavian artery supply disruption sequence: Hypothesis of a vascular etiology for Poland, Klippel-Feil and Möbius anomalies. Am J Med Genet, 1986; 23: 903-18

4. Cingel V, Bohac M, Mestanova V et al: Poland syndrome: from embryological basis to plastic surgery. Surg Radiol Anat, 2013; 35(8): $639-46$

5. Happle R: Poland anomaly may be explained as a paradominant trait. Am J Med Genet, 1999; 87: 364-65
6. van Steensel MA: Poland anomaly: Not unilateral or bilateral but mosaic. Am J Med Genet Part A, 2004; 125A: 211-12

7. Hidradenitis Suppurativa Foundation (Second congress, San Francisco March 2009), www.hs.foundation.org

8. Alikhan A, Lynch PJ, Eisen DB: Hidradenitis suppurativa: A comprehensive. J Am Acad Dermatol, 2009; 60(4): 539-61

9. Bhalla $R$, Sequeira W: Arthritis associated with hidradenitis suppurativa. Ann Rheum Dis, 1994; 53(1): 64-66

10. Vazirnia A, Cohen PR: Poland's syndrome: A concise review of the clinical features highlighting associated dermatologic manifestations. Am J Clin Dermatol, 2015; 16(4): 295-301

11. Erickson RP: Recent advances in the study of somatic mosaicism and diseases other than cancer. Curr Opin Genet Dev, 2014; 26: 73-78 